\title{
CONTROLLABILITY OF TIME-INVARIANT SINGULAR LINEAR SYSTEMS
}

\author{
M. Isabel García-Planas \\ Departament Matemàtica Aplicada I \\ Universitat Politècnica de Catalunya \\ Spain \\ maria.isabel.garcia@upc.edu
}

\author{
Sonia Tarragona \\ Departament Matemàtica Aplicada I \\ Universitat Politècnica de Catalunya \\ Spain \\ soniatarragona@hotmail.com
}

\begin{abstract}
We consider triples of matrices $(E, A, B)$, representing singular linear time invariant systems in the form $E \dot{x}(t)=A x(t)+B u(t)$, with $E, A \in M_{n}(C)$ and $B \in M_{n \times m}(C)$, under proportional and derivative feedback.

Structural invariants under equivalence relation characterizing singular linear systems are used to obtain conditions for controllability of the systems.
\end{abstract}

\section{Key words}

Singular systems, proportional and derivative feedback, controllability.

\section{Introduction}

We consider linear and time-invariant continuous singular systems of the form

$$
E \dot{x}(t)=A x(t)+B u(t), \quad x\left(t_{0}\right)=x_{0},
$$

where $E, A \in M_{n}(C), B \in M_{n \times m}(C)$, and $\dot{x}=$ $d x / d t$, that we will represent us a triples of matrices $(E, A, B)$, and we will denote by $M$, the set of this kind of triples.

Singular systems also called descriptor systems, generalized systems or diferential/algebraic systems, are found in engineering systems such as electrical, chemical processing circuit or power systems among others, and they have attracted interest in recent years.

In this paper, we present a collection of structural invariants which we will call controllability indices of the triple in terms of ranks of certain matrices associated to the triple, that permit us to give the explicit form of reduced triple $\left(I_{n}, A_{2}, B_{2}\right)$ without knowing the transformation matrices reducing the triple. As a corollary, a necessary and sufficient condition for controllability of the triple is deduced also in terms of the rank of a certain matrix.
We recall that the authors L. Dai [1], and W. Ratz [5] studied the controllability character for singular systems but they do not consider feedback and derivative feedback in the equivalence relation, only consider basis change in the state space, input space and premultiplication the system by invertible matrices.

The problem to obtain structural invariants permitting us to conclude conditions for controllability, was largely studied for standard linear systems under several equivalence relations that can be considered ([3],[4], [6] for example).

In the sequel we identify triples of matrices $(E, A, B)$ with rectangular matrices $(E A B)$ in order to use matrix expressions.

\section{Feedback equivalence}

Different useful and interesting equivalence relations between generalized systems have been defined. We deal with the equivalence relation $\left(E^{\prime}, A^{\prime}, B^{\prime}\right)=$ $\left(Q E P+Q B F_{E}, Q A P+Q B F_{A}, Q B R\right)$. with $P \in$ $G l(n ; C), Q \in G l(n ; C), R \in G l(m ; C), F_{A}, F_{E} \in$ $M_{m \times n}(C)$, that is to say the equivalence relation accepting one or more, of the following standard transformations: basis change in the state space, input space, feedback, derivative feedback and premultiplication by an invertible matrix.

Definition 1. Two triples $\left(E^{\prime}, A^{\prime}, B^{\prime}\right)$ and $(E, A, B)$ in $M$ are called equivalent if, and only if, there exist matrices $P \in G l(n ; C), Q \in G l(n ; C), R \in$ $G l(m ; C), F_{E}, F_{A} \in M_{m \times n}(C)$, such that

$$
\begin{aligned}
& \left(E^{\prime}, A^{\prime}, B^{\prime}\right)= \\
& \left(Q E P+Q B F_{E}, Q A P+Q B F_{A}, Q B R\right),
\end{aligned}
$$

or in a matrix form

$$
\left(\begin{array}{lll}
E^{\prime} & A^{\prime} & B^{\prime}
\end{array}\right)=Q\left(\begin{array}{lll}
E & A & B
\end{array}\right)\left(\begin{array}{ccc}
P & 0 & 0 \\
0 & P & 0 \\
F_{E} & F_{A} & R
\end{array}\right)
$$


It is easy to check that this relation is an equivalence relation.

Making use of the following notations.

- $I_{n}$ denotes the $n$-order identity matrix,

- $N$ denotes a nilpotent matrix in its reduced form $N=$ $\operatorname{diag}\left(N_{1}, \ldots, N_{\ell}\right), N_{i}=\left(\begin{array}{cc}0 & I_{n_{i}-1} \\ 0 & 0\end{array}\right) \in M_{n_{i}}(C)$,

- $J$ denotes the Jordan matrix $J=\operatorname{diag}\left(J_{1}, \ldots, J_{t}\right)$, $J_{i}=\operatorname{diag}\left(J_{i_{1}}, \ldots, J_{i_{s}}\right), J_{i_{j}}=\lambda_{i} I+N$,

- $L=\operatorname{diag}=\left(L_{1}, \ldots, L_{q}\right), L_{j}=\left(I_{n_{j}} 0\right) \in$ $M_{n_{j} \times\left(n_{j}+1\right)}(C)$,

- $R=\operatorname{diag}\left(R_{1}, \ldots, R_{p}\right), R_{n_{j}}=\left(0 I_{n_{j}}\right) \in$ $M_{n_{j} \times\left(n_{j}+1\right)}(C)$.

Theorem 1 ([2]). Let $(E, A, B)$ be a triple. Then, it is equivalent to

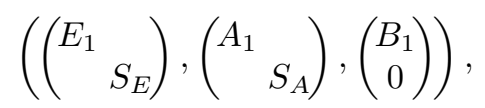

where $\left(E_{1}, A_{1}, B_{1}\right)$ is a regularizable triple in its Kronecker reduced form (see [2]), concretely

$$
\begin{aligned}
& \left(E_{1}, A_{1}, B_{1}\right)= \\
& \left(\left(\begin{array}{lll}
I_{1} & & \\
& I_{2} & \\
& & N_{2}
\end{array}\right),\left(\begin{array}{lll}
N_{1} & & \\
& J & \\
& & I_{3}
\end{array}\right),\left(\begin{array}{c}
B_{1} \\
0 \\
0
\end{array}\right)\right)
\end{aligned}
$$

The triple $\left(I_{1}, N_{1}, B_{1}\right)$, is a controllable standard system in its Kronecker reduced form, $\left(I_{2}, J, 0\right)$ corresponds to the finite zeros of the triple and $J$ in its Jordan reduced form, $\left(N_{2}, I_{3}, 0\right)$ corresponds to the infinite zeros of the triple and $N_{2}$ in its Jordan reduced form. The triple $\left(S_{E}, S_{A}, 0\right)$ is the strictly singular part of the system in its Kronecker reduced form:

$$
\left(\left(\begin{array}{lll}
L_{1} & & \\
& L_{2}^{t} & 0
\end{array}\right),\left(\begin{array}{lll}
R_{1} & & \\
& R_{2}^{t} & 0
\end{array}\right),\left(\begin{array}{l}
0 \\
0
\end{array}\right)\right)
$$

A complete system of invariants to obtain the canonical reduced form can be fond in [2].

\section{Controllability}

We recall that a system is called controllable (see [1]) if, for any $t_{1}>0, x(0) \in R^{n}$ and $w \in R^{n}$, there exists a control input $u(t)$ such that $x\left(t_{1}\right)=w$.

Equivalently

Theorem 2 ([1]). A system $(E, A, B) \in M$ is controllable if and only if

$$
\begin{aligned}
& \operatorname{rank}(E B)=n, \\
& \operatorname{rank}(s E-A B)=n, \text { for all } s \in C .
\end{aligned}
$$

First of all we prove that the controllability is preserved by the equivalence relation considered.
Proposition 1. The controllability character is invariant under equivalence relation considered.

Proof. Let $(E, A, B), \quad\left(E^{\prime}, A^{\prime}, B^{\prime}\right)$ two equivalent triples. So, there exist matrices $Q, P \in G l(n ; C)$, $R \in G l(m ; C)$ and $F_{E}, F_{A} \in M_{m \times n}(C)$ such that

$$
\left(\begin{array}{lll}
E^{\prime} & A^{\prime} & B^{\prime}
\end{array}\right)=Q\left(\begin{array}{lll}
E & A & B
\end{array}\right)\left(\begin{array}{ccc}
P & 0 & 0 \\
0 & P & 0 \\
F_{E} & F_{A} & R
\end{array}\right) .
$$

Then

$$
\begin{aligned}
& \operatorname{rank}\left(\begin{array}{ll}
E^{\prime} & B^{\prime}
\end{array}\right)=\operatorname{rank} Q\left(\begin{array}{ll}
E & B
\end{array}\right)\left(\begin{array}{c}
P \\
F_{E}
\end{array}\right)= \\
& \operatorname{rank}(E B)
\end{aligned}
$$

and

$$
\begin{aligned}
& \operatorname{rank}\left(s E^{\prime}-A^{\prime} B^{\prime}\right)= \\
& \operatorname{rank} Q(s E-A B)\left(\begin{array}{c}
P \\
s F_{E}-F_{A} R
\end{array}\right)= \\
& \operatorname{rank}(s E-A B) .
\end{aligned}
$$

This proposition permit us if necessary, to take an equivalent triple in its canonical reduced form.

Proposition 2. A necessary condition for controllability is that the system be standardizable. That is to say, the triple is equivalent to $\left(I, N, B_{1}\right)$.

Proof. It suffices to take a triple $(E, A, B)$ in its reduced form and compute $\operatorname{rank}(E B)$, and rank $(s E-A B)$.

We consider the following matrices

$$
\begin{aligned}
& M_{i}(C) \in M_{(i+1) n \times(i n+2 i m)}(C) . \\
& M_{1}=\left(\begin{array}{ccc}
E & B & 0 \\
A & 0 & B
\end{array}\right) \text {, } \\
& M_{2}=\left(\begin{array}{cccccc}
E & B & 0 & 0 & 0 & 0 \\
A & 0 & B & E & B & 0 \\
0 & 0 & 0 & A & 0 & B
\end{array}\right) \text {, } \\
& M_{3}=\left(\begin{array}{ccccccccc}
E & B & 0 & 0 & 0 & 0 & 0 & 0 & 0 \\
A & 0 & B & E & B & 0 & 0 & 0 & 0 \\
0 & 0 & 0 & A & 0 & B & E & B & 0 \\
0 & 0 & 0 & 0 & 0 & 0 & A & 0 & B
\end{array}\right), \\
& M_{\ell}=\left(\begin{array}{llllllllll}
E & B & 0 & 0 & 0 & 0 & 0 & 0 & 0 & \\
A & 0 & B & E & B & 0 & 0 & 0 & 0 & \\
0 & 0 & 0 & A & 0 & B & E & B & 0 & \\
0 & 0 & 0 & 0 & 0 & 0 & A & 0 & B & \\
& & & & & & & & & \ddots
\end{array}\right) .
\end{aligned}
$$

Definition 2. We consider the following numbers $r=$ $\left(r_{1}, \ldots, r_{\ell}, \ldots\right)$, where $r_{i}=\operatorname{rank} M_{i}, \forall i=1,2, \ldots$ 
Proposition 3. In the set $M$ of singular systems, the $r_{i}$ numbers are invariant under the equivalence relation considered.

Proof. Let $(E, A, B),\left(E^{\prime}, A^{\prime}, B^{\prime}\right)$ be two equivalent triples in $M$, then, there exist matrices $P, Q \in$ $G l(n ; C), R \in G l(m ; C), F_{E}, F_{A} \in M_{m \times n}(C)$ such that

$$
\left(\begin{array}{lll}
E^{\prime} & A^{\prime} & B^{\prime}
\end{array}\right)=Q\left(\begin{array}{lll}
E & A & B
\end{array}\right)\left(\begin{array}{ccc}
P & 0 & 0 \\
0 & P & 0 \\
F_{E} & F_{A} & R
\end{array}\right)
$$

So,

$$
\begin{aligned}
& r_{1}{ }^{\prime}=\operatorname{rank}\left(\begin{array}{lll}
E^{\prime} & B^{\prime} & 0 \\
A^{\prime} & 0 & B^{\prime}
\end{array}\right)= \\
& =\operatorname{rank}\left(\begin{array}{ll}
Q & 0 \\
0 & Q
\end{array}\right)\left(\begin{array}{ccc}
E & B & 0 \\
A & 0 & B
\end{array}\right)\left(\begin{array}{ccc}
P & 0 & 0 \\
F_{E} & R & 0 \\
F_{A} & 0 & R
\end{array}\right)= \\
& =\operatorname{rank}\left(\begin{array}{lll}
E & B & 0 \\
A & 0 & B
\end{array}\right)=r_{1} .
\end{aligned}
$$$$
\text { Calling } \mathbf{Q}=\left(\begin{array}{lll}
Q & & \\
& \ddots & \\
& & Q
\end{array}\right) \text { and }
$$$$
\mathbf{P}=\left(\begin{array}{ccccccccc}
P & 0 & 0 & & & & & \\
F_{E} & R & 0 & & & & & \\
F_{A} & 0 & R & & & & & \\
& & & P & 0 & 0 & \\
& & F_{E} & R & 0 & & \\
& & & F_{A} & 0 & R & \\
& & & & & & \ddots
\end{array}\right)
$$$$
r_{\ell}^{\prime}=\operatorname{rank}\left(\begin{array}{ccccccc}
E^{\prime} & B^{\prime} & 0 & 0 & 0 & 0 & \\
A^{\prime} & 0 & B^{\prime} & E^{\prime} & B^{\prime} & 0 & \\
0 & 0 & 0 & A^{\prime} & 0 & B^{\prime} & \\
& & & & & & \ddots
\end{array}\right)=
$$$$
=\operatorname{rank}=\mathbf{Q}\left(\begin{array}{ccc}
E & B & 0 \\
A & 0 & B
\end{array}\right) \mathbf{P}=
$$$$
=\operatorname{rank}\left(\begin{array}{ccccccc}
E & B & 0 & 0 & 0 & 0 & \\
A & 0 & B & E & B & 0 & \\
0 & 0 & 0 & A & 0 & B & \\
& & & & & & \ddots
\end{array}\right)=r_{\ell}
$$

Theorem 3. A triple $(E, A, B)$ is controllable if and only if $r_{n-1}=n^{2}$.

Proof. Suppose now that the triple is controllable, taking into account proposition 2 , the triple is equivalent to an standard one $\left(I, N, B_{1}\right)$. Then, computing $r_{n-1}$ in this equivalent reduced form, we obtain

$$
r_{n-1}=(n-1) n+\operatorname{rank}\left(B_{1} N B_{1} \ldots N^{n-1} B_{1}\right) .
$$

We observe that $r_{n-1}=n^{2}$ if and only if $\left(N, B_{1}\right)$ is controllable.

Conversely, suppose $r_{n-1}=n^{2}$, we have rank $(E B)=n$, because $(E B)$ correspond to the first $n$-row block matrix in the $n^{2} \times((n-1) n+2(n-1) m)$ matrix $\left(\begin{array}{ccccccc}E & B & 0 & 0 & 0 & 0 & \\ A & 0 & B & E & B & 0 & \\ 0 & 0 & 0 & A & 0 & B & \\ & & & & & & \ddots\end{array}\right)$ having full rank. So the triple is standardizable and we can compute $r_{n-1}$ using the reduced form $\left(I_{n}, A_{1}, B_{1}\right)$. Finally, it suffices to observe that the standardizable triple $(E, A, B)$ is controllable if and only if $\left(A_{1}, B_{1}\right)$ is controllable:

$\operatorname{rank}(E B)=\operatorname{rank}\left(I_{n} B_{1}\right)=n$,

$\operatorname{rank}(s E-A B)=\operatorname{rank}\left(s I_{n}-A_{1} B_{1}\right)=n, \forall s \in C$.

Finally, we define a collection of numbers that permit us to deduce the controllability indices of a controllable triple.

We call $r_{0}=\operatorname{rank} B$, and we define the $\rho$-numbers in the following manner.

\section{Definition 3.}

$$
\begin{aligned}
\rho_{0} & =r_{0} \\
\rho_{1} & =r_{1}-r_{0}-n \\
\rho_{2} & =r_{2}-r_{1}-n \\
\vdots & \\
\rho_{s} & =r_{s-1}-r_{s}-n .
\end{aligned}
$$

It is obvious the following proposition.

Proposition 4. The $\rho$-numbers are invariant under equivalence relation considered.

Proposition 5. The controllability indices $\left[k_{1}, \ldots, k_{p}\right]$ of a controllable triple, are the conjugate partition of $\left[\rho_{0}, \rho_{1}, \ldots, \rho_{s}\right]$.

Proof. It suffices to compute the $\rho$-numbers of a equivalent reduced form to the controllable triple.

We observe that if the $(E, A, B)$ is controllable then $k_{1}+\ldots+k_{p}=n$ and $p=\rho_{0}=\operatorname{rank} B$.

Theorem 4. Let $(E, A, B)$ be a controllable triple with Kronecker indices $\left(k_{1}, \ldots, k_{p}\right)$. Then the triple can be reduced to $\left(I_{n}, A_{1}, B_{1}\right)$ with

$$
A_{1}=\left(\begin{array}{ccc}
N_{1} & & \\
& \ddots & \\
& & N_{p}
\end{array}\right), B_{1}=\left(\begin{array}{ccc}
B_{1}^{1} & & \\
& \ddots & \\
& & B_{p}^{1}
\end{array}\right)
$$

and $N_{i}=\left(\begin{array}{cccc}0 & 1 & & \\ & \ddots & \ddots & \\ & & & 1 \\ & & & 0\end{array}\right) \in M_{k_{i}}(C), B_{i}^{1}=\left(\begin{array}{c}0 \\ \vdots \\ 0 \\ 1\end{array}\right) \in$ $M_{k_{i} \times 1}(C)$. 
Proof. It suffices to observe that the Kronecker indices for $(E, A, B)$ coincide with Kronecker of the pair $\left(A_{1}, B_{1}\right)$.

Example 1. 1) Let $(E, A, B)$ be a triple with $E=$ $\left(\begin{array}{llll}2 & 1 & 1 & 1 \\ 1 & 2 & 1 & 1 \\ 2 & 3 & 3 & 2 \\ 0 & 1 & 0 & 2\end{array}\right), A=\left(\begin{array}{cccc}1 & 2 & 1 & 1 \\ 1 & 1 & 2 & 0 \\ 3 & 2 & 1 & -1 \\ 2 & 1 & 3 & 5\end{array}\right), B=\left(\begin{array}{ll}0 & 0 \\ 0 & 0 \\ 5 & 0 \\ 0 & 2\end{array}\right)$.

Computing the $r_{i}$ numbers we obtain, $r_{0}=2, r_{1}=7$, $r_{2}=12, r_{3}=16=r_{2}+n$. Then $\rho_{0}=2, \rho_{1}=1$, $\rho_{2}=1$ and the Kronecker indices are $k_{1}=3, k_{2}=1$. So $(E, A, B) \sim\left(I_{4}, A_{1}, B_{1}\right)$ with $A_{1}=\left(\begin{array}{llll}0 & 1 & 0 & 0 \\ 0 & 0 & 1 & 0 \\ 0 & 0 & 0 & 0 \\ 0 & 0 & 0 & 0\end{array}\right)$, $B_{1}=\left(\begin{array}{ll}0 & 0 \\ 0 & 0 \\ 1 & 0 \\ 0 & 1\end{array}\right)$

2) Let $(E, A, B)$ be a triple with $E=\left(\begin{array}{llll}3 & 1 & 1 & 1 \\ 1 & 3 & 1 & 1 \\ 1 & 1 & 3 & 1 \\ 1 & 1 & 1 & 3\end{array}\right), A=$ $\left(\begin{array}{llll}0 & 3 & 0 & 1 \\ 0 & 1 & 0 & 1 \\ 0 & 1 & 0 & 3 \\ 0 & 1 & 0 & 1\end{array}\right), B=\left(\begin{array}{ll}1 & 1 \\ 3 & 1 \\ 1 & 1 \\ 1 & 3\end{array}\right)$

Computing the $r_{i}$ numbers we obtain, $r_{0}=2, r_{1}=8$, $r_{2}=12=r_{1}+n$. Then $\rho_{0}=2, \rho_{1}=2$ and the Kronecker indices are $k_{1}=2, k_{2}=2$. So $(E, A, B) \sim$ $\left(I_{4}, A_{1}, B_{1}\right)$ with $A_{1}=\left(\begin{array}{llll}0 & 1 & 0 & 0 \\ 0 & 0 & 0 & 0 \\ 0 & 0 & 0 & 1 \\ 0 & 0 & 0 & 0\end{array}\right), B_{1}=\left(\begin{array}{ll}0 & 0 \\ 1 & 0 \\ 0 & 0 \\ 0 & 1\end{array}\right)$.

\section{References}

L. Dai "Singular Control Systems". Springer Verlag. New York (1989).

Mํㅡ. I. García-Planas, A Complete system of structural invariants for singular systems under proportional and derivative feedback To appear in Int. J. Contemp. Math. Scinces. (2009)

Ma I. García-Planas, M.D. Magret, An alternative System of Structural Invariants for Quadruples of Matrices, Linear Algebra and its Applications 291, (1-3), pp. 83-102 (1999).

A.S. Morse, Structural invariants of linear multivariable systems, SIAM J. Contr. 11, pp. 446-465 (1973). W. Ratz Canonical forms for linear descriptor systems with variable coefficients. Treihe der Chemnitzer DFG-Forschergruppe Scientific Parallel Computing, Chemnitz, Germany (1996).

J. S. Thorp, The Singular Pencil of Linear Dynamical System, System. Int. J. Control, 18 (3) (1973), pp. 557-596. 